\section{Neumonía lipoidea exógena: Una causa inhabitual de nódulos pulmonares Casos clínicos}

\author{
FELIPE ALIAGA ${ }^{1, \mathrm{a}}$, SARA CHERNILO ${ }^{2}$, CRISTINA FERNÁNDEZ $^{3}$, \\ HUGO VALENZUELA ${ }^{2}$, JUAN CARLOS RODRÍGUEZ ${ }^{2}$
}

\section{Exogenous lipoid pneumonia. Report of three cases}

Lipoid pneumonia is an unusual cause of aspiration pneumonia with diverse radiologic manifestations. One of these are pulmonary nodules in which the main differential diagnosis is pulmonary carcinoma. We report an 85 years old male, an 85 years old female and a 34 years old male in whom percutaneous biopsies of suspicious nodules were compatible with lipoid pneumonia.

(Rev Med Chile 2017; 145: 1495-1499)

Key words: Pneumonia, Aspiration; Pneumonia, Lipid; Solitary Pulmonary Nodule.

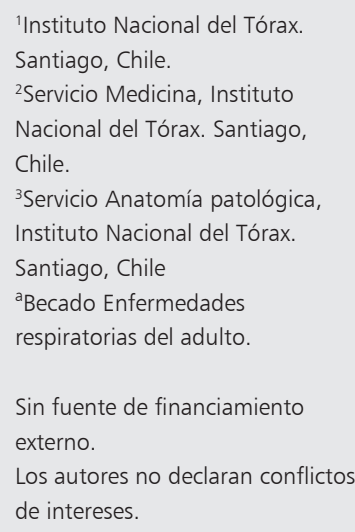

Recibido el 8 de octubre de 2017 , aceptado el 18 de diciembre de 2017

Correspondencia a: Felipe Aliaga Tapia, Llewellyn Jones 1539, Providencia, Santiago. felipealiaga@gmail.com
L a neumonía lipoidea exógena (NL) es una forma infrecuente de neumonía aspirativa de lípidos y de macrófagos cargados con éstos.

Para el diagnóstico generalmente se requiere confirmación histopatológica, dado lo inespecífico de su cuadro clínico. En ocasiones puede presentarse en forma de lesiones nodulares, que obligan a un diagnóstico diferencial amplio.

Presentamos 3 casos clínicos de neumonía lipoidea, confirmados histológicamente, que se presentaron inicialmente como nódulos pulmonares.

\section{Caso 1}

Hombre de 85 años con antecedentes de enfermedad de Chagas un megacolon operado y un cáncer de próstata tratado. Previo a su colectomía usaba vaselina para la constipación.

En el contexto de un cuadro de tipo gripal se realizó una radiografía de tórax que demostró condensaciones en ambas bases pulmonares. Los exámenes de laboratorio habitual no mostraban alteraciones. La tomografía axial computarizada (TAC) de tórax (Figura 1 A y B) reveló múltiples nódulos y masas pulmonares de bordes irregulares, algunas cavitadas y con necrosis. La TAC de cavidades perinasales fue normal. El estudio reumatológico con ANA, FR y ANCA fue negativo y la evaluación por reumatología descartó una vasculitis. Posteriormente se realizó una fibrobroncoscopía con lavado broncoalveolar (LBA) y biopsia transbronquial que resultaron no diagnósticos. Una biopsia tomada mediante una punción percutánea, reveló parénquima pulmonar con espacios alveolares discretamente dilatados y acúmulos de macrófagos con fagocitosis de material lipídico concordante con neumonía lipoidea (Figura 2).

No se indicó tratamiento específico, se le sugirió no volver a utilizar vaselina o similares. El paciente se mantiene asintomático y en control periódico.

\section{Caso 2}

Mujer de 85 años, no fumadora con antecedentes de una cirugía resectiva del maxilar por un carcinoma escamoso y el uso frecuente de mentholatum para su congestión nasal habitual. 


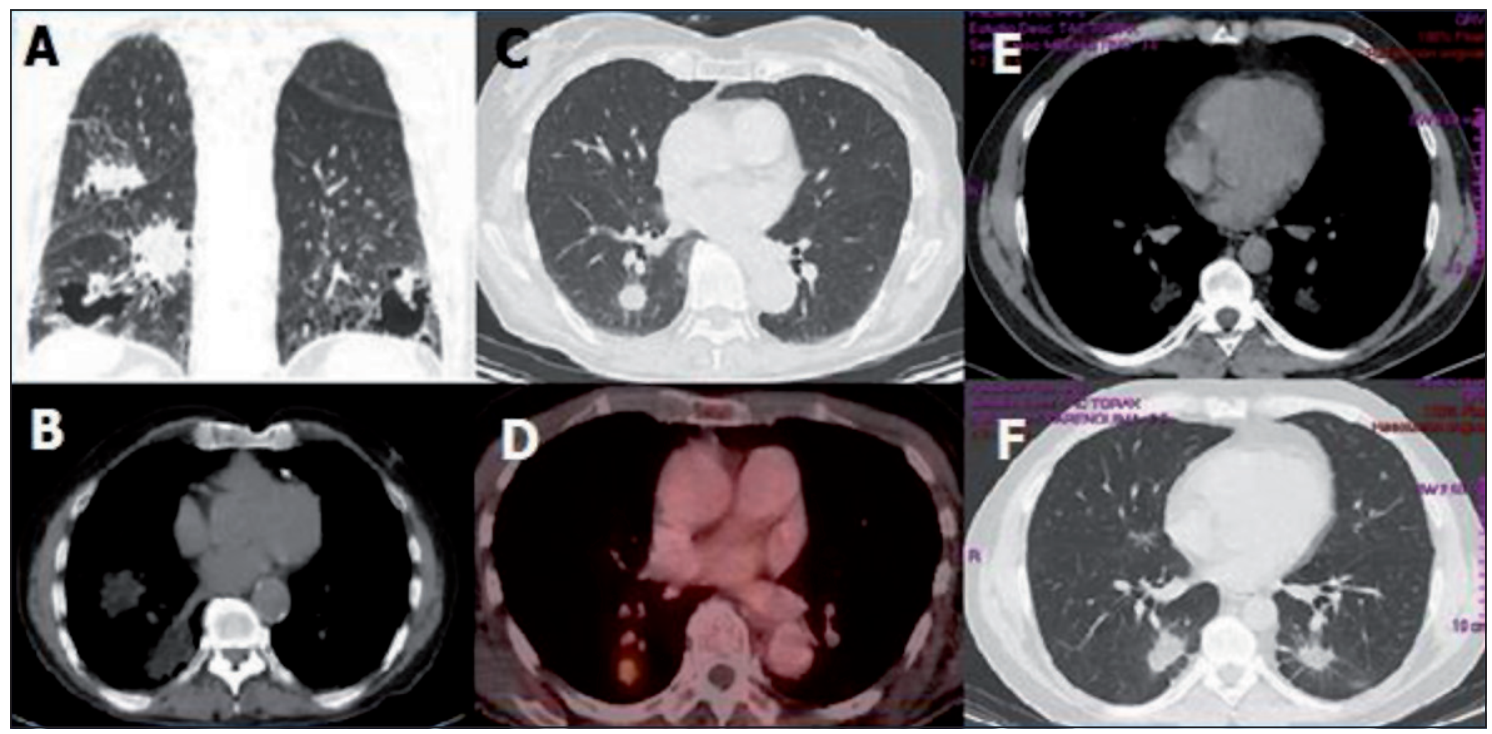

Figura 1. Estudio imagenológico en 3 casos de neumonía lipoidea. A: TAC corte coronal, caso 1: Nódulos y masas bilaterales de contornos mal definido algunos de ellos cavitados. B: TAC corte axial, técnica de mediastino, caso 1: Nódulos pulmonares con áreas de baja atenuación compatibles con densidad de tejidos grasos. C: TAC corte axial, caso 2: Nódulo de 17 mm espiculado en lóbulo inferior derecho. D: PET/CT, caso 2: Nódulo antes descrito con aumento de captación de radiofármaco en forma significativa (SUV Max 3.9). E: TAC técnica mediastino, corte axial, caso 3: Nódulos con densidad de tejido graso en ambos lóbulos inferiores. F: TAC corte axial caso 3: Nódulos espiculados en lóbulos inferiores.

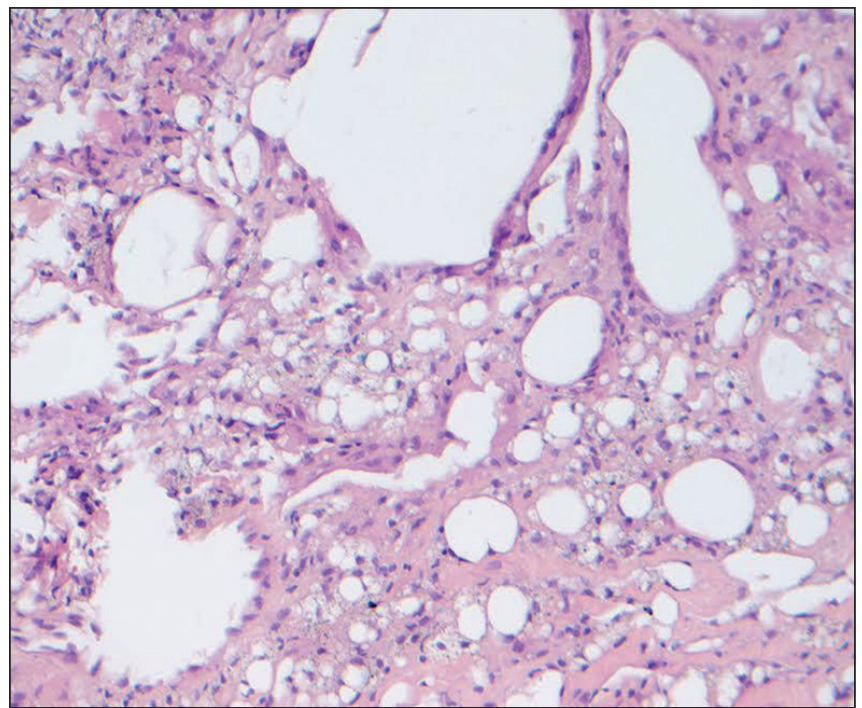

Figura 2. Biopsia pulmonar obtenida mediante punción percutánea guiada por TAC correspondiente al paciente número 1 (HE 40X): Se identifica parénquima pulmonar con desarrollo de espacios discretamente dilatados con acúmulos de macrófagos con fagocitosis de material lipídico concordante con neumonía de tipo lipoidea.
Durante el seguimiento oncológico se realizó un PET/CT (Figura 1 C y D) que demostró un nódulo de bordes espiculados de $17 \mathrm{~mm}$ en el lóbulo inferior derecho con SUV Max 3,9. En este contexto lo más importante era descartar una metástasis de su tumor maxilar.
Se realizó una punción percutánea bajo TAC que demostró parénquima pulmonar de arquitectura distorsionada, con numerosos histiocitos y células gigantes multinucleadas, que presentaban gotas lipídicas de distinto tamaño en su citoplasma. El intersticio presentaba fibrosis reparativa 
acompañada de focos inflamatorios de tipo crónico. Estos hallazgos son compatibles con neumonía lipoidea crónica.

No se indicaron terapias específicas. Se le sugirió no usar más mentholatum. Y se repitió el TAC a los 6 meses y éste no presentaba cambios.

\section{Caso 3}

Hombre de 34 años, fumador. Consultó por dolor en hemitórax derecho, realizándose primero una radiografía de tórax y luego una TAC que reveló nódulos y masas espiculadas en ambas bases pulmonares, la mayor de ellas de $40 \mathrm{~mm}$ (Figura 1, E y F). Se realizó una punción percutánea que fue compatible con neumonía lipoidea exógena. Luego de esto el paciente fue reinterrogado manifestando el uso de aceite gomenolado intranasal durante 3 años para alivio de la congestión nasal. Durante 10 años de seguimiento no ha presentado molestias y las imágenes en la TAC no han variado.

\section{Discusión}

Existen múltiples complicaciones pulmonares causadas por la aspiración de distintas sustancias hacia la vía aérea. La NL es una condición poco común resultante de la aspiración y acumulación de lípidos de origen animal, vegetal o mineral en el tejido pulmonar. Puede ser clasificada como endógena si el material depositado proviene del mismo pulmón y de origen exógeno cuando el material aspirado proviene del exterior.

La NL fue descrita por primera vez en 1925 por Laughlen asociada al uso de laxantes ${ }^{1}$. Desde entonces se la ha denominado de distintas formas: neumonía de colesterol, parafinoma y granulomatosis lipídica, y neumonía lipoidea término que es hoy día el más aceptado. Se asocia a múltiples sustancias, como aceites minerales, vegetales, vaselina, jalea de petróleo y otras menos frecuentes como exposición laboral a parafina, insecticida, pinturas en aerosol e incluso en artistas escupefuegos que utilizan gasolina o kerosene para ello $^{2-5}$. En adultos lo más frecuente es que sea secundario al uso de lubricantes utilizados como laxantes o lubricantes nasales o labiales. En nuestros tres casos existía este antecedente. En el primero el paciente usó por varios años vaselina en cápsulas para tratar su constipación crónica secundaria a su enfermedad de Chagas. En el segundo la paciente utilizaba mentholatum intranasal, cuya base es la vaselina, un hidrocarburo de origen mineral. El tercero usaba aceite gomenolado como lubricante nasal.

En su patogenia es primordial la exposición a algunas de las substancias mencionadas, las que suelen inhibir el reflejo de la tos lo que facilita la aspiración. A esto habitualmente se asocia algún trastorno de la deglución, dentro de los que destacan las enfermedades neurológicas, la fístula gastro esofágica, una hernia hiatal u otros como la enfermedad de Chagas en que se combina por un lado la constipación crónica secundaria al megacolon y el factor de riesgo aspirativo dado por el compromiso esofágico, como el caso número $1^{6}$. Los aceites aspirados llegan a los alvéolos donde son fagocitados por los macrófagos los que incapaces de metabolizarlos, rompen su pared con liberación de los ácidos grasos produciendo una reacción inflamatoria de células gigante a cuerpo extraño con inflamación crónica pudiendo llegar a la fibrosis alvéolo-intersticial si el proceso se perpetúa ${ }^{7}$.

Desde el punto de vista clínico los síntomas varían según la cantidad y el tipo de sustancia inhalada, pero casi siempre la clínica es inespecífica y se manifiesta por tos o una disnea leve, y no infrecuentemente es un hallazgo radiológico.

Ocasionalmente existen casos en que se inhalan grandes cantidades de sustancias altamente irritantes que pueden producir falla respiratoria aguda grave con fiebre e inflamación sistémica asociada, con necesidad de asistencia ventilatoria, incluso con reporte de casos fatales ${ }^{8}$.

Las manifestaciones radiológicas son variadas por lo que la NL debería incluirse en el diagnóstico diferencial de múltiples enfermedades, como el cáncer broncogénico, metástasis, neumonías infecciosas, nódulos reumatoides, poliangeitis con granulomatosis, entre otros ${ }^{9}$.

Los hallazgos más comunes son: el aumento de la densidad radiológica con patrón de vidrio esmerilado, la condensación, el patrón en empedrado, y los nódulos o masas. Las consolidaciones son habitualmente heterogéneas y al ser evaluadas con técnica de mediastino presentan densidades negativas (densidad grasa) entre - 30 y - $150 \mathrm{UH}$ lo que puede ser de utilidad para el diagnóstico ${ }^{10}$. En nuestros casos la radiología reveló nódulos, uno de ellos cavitado (caso 1) y los otros sólidos. Fue- 
ron considerados inicialmente como neoplásicos aunque con densidad de tejido graso.

Con respecto al PET/CT están documentados casos de falsos positivos para neoplasia al existir actividad inflamatoria local ${ }^{11}$. En el caso 2 se realizó un PET/CT que demostró aumento de la captación en forma significativa lo que planteó como primera posibilidad una metástasis.

El diagnóstico se basa en una historia concordante con el antecedente de exposición a agentes productores de NL, asociado a la comprobación ya sea de macrófagos cargados con material lipídico en el LBA o estudio histopatológico pulmonar característico. El LBA puede sugerir el diagnóstico ante el hallazgo de un líquido de apariencia blanquecino turbio con una interfase con gotas de grasa en su superficie y contenido acuoso en la parte inferior, aunque el hallazgo más sugerente es la identificación de macrófagos alveolares cargados con lípidos ${ }^{12}$. Muchas veces es necesaria la biopsia pulmonar, que puede realizarse ya sea por fibrobroncoscopía, o por punción percutánea. La biopsia quirúrgica no suele ser necesaria ${ }^{13}$. En la anatomía patológica al utilizar las técnicas clásicas de fijación con parafina los lípidos son disueltos por el xilol por lo que se observan "espacios vacíos" que representan el material graso disuelto formando vacuolas. Además se observan macrófagos cargados con lípidos que rellenan y distienden los alvéolos y el intersticio, pudiendo asociarse a inflamación y fibrosis. Con respecto a los casos expuestos sólo en uno de ellos se realizó LBA el cual no fue sugerente de NL, por lo que fue necesario realizar estudio histológico con punción percutánea, procedimiento que fue diagnóstico en los tres casos.

Con respecto a las complicaciones asociadas se ha comunicado una mayor incidencia de infección por micobacterias ambientales y nocardia ${ }^{14}$. Esto explicado aparentemente porque los lípidos propician su crecimiento y alteran la fagocitosis del huésped. Otras complicaciones descritas son el desarrollo de fibrosis pulmonar, insuficiencia respiratoria y cor pulmonale, pero son excepcionales.

Con respecto al tratamiento no existen estudios que definan la mejor opción terapéutica, aunque la primera medida a adoptar es identificar y suspender el agente causal ${ }^{15}$ lo que habitualmente es suficiente, sobre todo si el paciente no presenta síntomas. Existen algunos reportes que sugieren que el uso de corticoides reduciría la inflamación, pero éstos se reservan sólo para los casos agudos en que exista daño pulmonar severo ${ }^{16}$. Otra opción terapéutica descrita es el uso de lavado pulmonar total de forma similar a lo que se realiza en la proteinosis alveolar ${ }^{17,18}$.

Por último, si existieran bronquiectasias asociadas se recomienda manejo médico reservando el tratamiento quirúrgico en los casos que no respondan a éste.

\section{Referencias}

1. Laughlen GF. Studies on pneumonia following naso-pharyngeal injection of oil. Am J Pathol 1925; 1 (4): 407-14.

2. Chauveau R, Medart L, Ghaye B. La pneumonie lipidique exogene: un diagnostic simple? Rev Med Liege 2005; 60: 10: 799-804.

3. Gondouin A, Manzoni P, Ranfaing E, Brun J, Cadranel J, Sadoun D, et al. Exogenous lipid pneumonia: a retrospective multicentre study of 44 cases in France. Eur Respir J 1996; 9: 1463-9.

4. Hadda V, Khilnani G. Lipoid pneumonia: an overview, Expert Review of Respiratory Medicine 2010; 4: 6: 799807.

5. Gentina T, Tillie-Leblond I, Birolleau S, Saidi F, Saelens T, Boudoux L, et al. Fire-eater's lung: seventeen cases and a review of the literature. Medicine (Baltimore) 2001; 80: 291-7.

6. García S, Aranha A, García F, Basile F, Pinto M, Oliveira E, et al. A retrospective study of histopathological findings in 894 cases of megacolon. What is the relationship between megacolon and colonic cancer? Rev Inst Med Trop S Paulo 2003; 45 (2): 91-3.

7. Parameswaran K, Anvari M, Efthimiadis A, Kamada D, Hargreave FE, Allen CJ. Lipid-laden macrophages in induced sputum are a marker of oropharyngeal reflux and possible gastric aspiration. Eur Respir J 2000; 16: 1119-22.

8. Requena-Kassarjlan Y. An infant with respiratory distress. Clin Pediatr 2001; 40: 507-9.

9. Marchiori E, Zanetti G, Mano CM, Irion KL, Daltro PA, Hochhegger B. Lipoid pneumonia in 53 patients after aspiration of mineral oil: comparison of high-resolution computed tomography findings in adults and children. J Comput Assist Tomogr 2010; 34 (1): 9-12.

10. Betancourt S, Martínez-Jiménez S, Rossi S, Truong M, Carrillo J, Erasmus J. Lipoid pneumonia: spectrum of clinical and radiologic manifestations. AJR Am J Roentgenol 2010; 194: 103-9. 
11. Fox BD, Shechtman I, Shitrit D, Bendayan D, Kramer MR. A "fat chance" it's malignant: lipoid pneumonia simulating lung cancer on PET scan. Thorax 2007; 62 (5): 464.

12. Lauque D, Dongay G, Levade T, Caratero C, Carles P. Bronchoalveolar lavage in liquid paraffin pneumonitis. Chest 1990; 98: 1149-55.

13. Kameswaran M, Annobil SH, Benjamin B, Salim M. Bronchoscopy in lipoid pneumonia. Arch Dis Child 1992; 67: 1376-7.

14. Ridaura-Sanz C, López-Corella E, Salazar-Flores M. Exogenous lipoid pneumonia superinfected with acidfast bacilli in infants: a report of nine cases. Fetal Pediatr Pathol 2006; 25: 107e17.
15. Baron SR, Haramati LD, Riviera VT. Radiological and clinical findings in acute and chronic exogenous lipoid pneumonia. Journal Thorac Imaging 2003; 18 : 217-24.

16. Ayvasian F, Steward D, Merkel C, Frederick W. Diffuse lipoid pneumonitis successfully treated with prednisone. Am J Med 1966; 43: 930-4.

17. Sias SM, Daltro PA, Marchiori E, Ferreira AS, Caetano RL, Silva CS, et al. Clinic and radiological improvement of lipoid pneumonia with multiple bronchoalveolar lavages. Pediatr Pulmonol 2009; 44: 309-15.

18. Lauque D, Dongay G, Levade T, Caratero C, Carles P. Bronchoalveolar lavage in liquid paraffin pneumonitis. Chest 1990; 98: 1149e55.

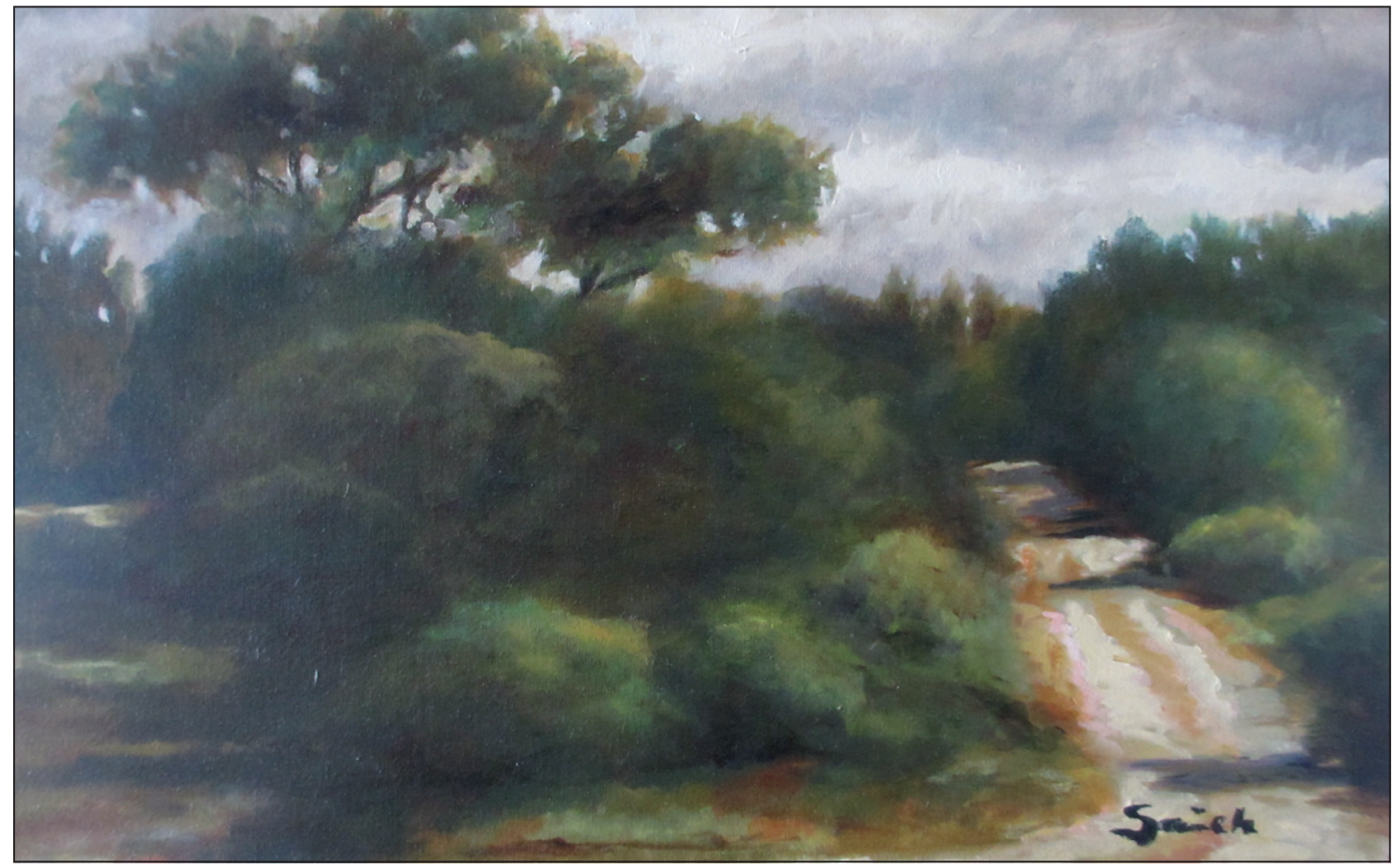

Caminito. Dr. Carlos Saieh Andonie 